\title{
ОБ ОТСУТСТВИИ ГЛОБАЛЬНЫХ РЕШЕНИЙ СМЕШАННОЙ ЗАДАЧИ ДЛЯ НЕЛИНЕЙНОГО ЭВОЛЮЦИОННОГО УРАВНЕНИЯ ТИПА ГИНЗБУРГА-ЛАНДАУ
}

\author{
ШI. М. Насибов \\ Представлено академиком РАН В.П. Масловым 16.07.2018 г. \\ Поступило 31.07.2018 г.

\begin{abstract}
Исследуется вопрос об отсутствии глобальных решений первой смешанной задачи для одного нелинейного эволюционного уравнения типа Гинзбурга-Ландау. Доказано, что при “достаточно больших начальных данных” отсутствуют глобальные решения исследуемой задачи.
\end{abstract}

Ключевые слова: нелинейное эволюционное уравнение, уравнение типа Гинзбурга-Ландау, смешанная задача, глобальное решение, отсутствие глобального решения.

DOI: https://doi.org/10.31857/S0869-56524842147-149

1. Пусть $\Omega \subset R^{n}-$ произвольная ограниченная область с гладкой границей. Рассмотрим следующую смешанную задачу:

$$
\begin{gathered}
u_{t}=(\alpha+i \beta) \Delta u+f(u, \nabla u), x \in \Omega, t>0, \\
u(x, 0)=u_{0}(x), x \in \bar{\Omega},\left.u(x, t)\right|_{\partial \Omega}=0, t \geq 0,
\end{gathered}
$$

Здесь

$$
f(u, \nabla u) \geq \omega_{1}|u|^{1+\gamma}+\omega_{2}|\nabla u|^{1+\mu},
$$

где $\omega_{1} \geq 0, \omega_{2} \geq 0, \omega_{1}^{2}+\omega_{2}^{2} \neq 0, \gamma>0, \mu>0, \beta \neq 0, \alpha \in R$.

Уравнение (1) встречается в различных разделах прикладной физики, в нелинейной квантовой механике, в теории распространения световых волн в нелинейных средах (см., например, [1-3]). При

$$
f(u)=i \gamma|u|^{p} u+i \mu u, \gamma \mu>0, p n \geq 4
$$

соответственно вопрос о разрушении решений задачи (1), (2) при $\alpha=0$ рассматривался в случае $\mu=0$ в [4], в случае $\mu>0$ в [5], а решений задачи Коши для уравнения (1) при $\mu=0, \alpha=0$ в [4, 6-10] и др. Для близких уравнений к (1), т.е. $f(u)=|u|^{1+p}, p>0$, вопросы разрушения решений задачи (1), (2) исследованы в [11, 12].

Задача Коши для уравнения (1) при $\alpha>0$ и $f(u, \nabla u)=F(|u|) u$ при подходящих условиях на $F(|u|)$ исследована в работах $[13,14]$ и др.

2. Обозначения и формулировка основных результатов. Пусть $\lambda_{1}-$ первое соб-

\section{Институт прикладной математики}

Бакинского государственного университета, Азербайджан

E-mail: nasibov_sharif@hotmail.com ственное число, $\vartheta_{1}(x)-$ соответствующая первая собственная функция задачи

$$
\Delta \vartheta+\lambda \vartheta=0, x \in \Omega ; \vartheta(x)=0, x \in \partial \Omega .
$$

Известно, что $\lambda_{1}>0, \vartheta_{1}(x)>0, \forall x \in \Omega$ (см., например, [15, c. 434]. Не умаляя общности, будем считать, что

$$
\int_{\Omega} \vartheta_{1}(x) d x=1
$$

Примем следующие обозначения:

$$
\omega=\omega_{1}+\omega_{0} \omega_{2},
$$

здесь

$$
\omega_{0}=\left[k_{0}^{-1}\left(\int_{\Omega} \vartheta_{1}^{-\frac{1}{\mu}}(x) d x\right)^{-\frac{\mu}{(1+\mu)}}\left\|\vartheta_{1}\right\|_{n}^{-1}\right]^{1+\mu} \text {, }
$$

где

$$
k_{0}=\frac{1}{n}\left(\frac{1}{\sigma_{n}}\right)^{\frac{1}{n}}, \sigma_{n}=\frac{\pi^{\frac{n}{2}}}{\Gamma\left(\frac{n}{2}+1\right)},
$$

$\Gamma(\cdot)$ - гамма-функция Эйлера. Здесь $\|\cdot\|_{p}$ - норма в $L_{p}(\Omega), p \geq 1$.

$$
\rho=\left\{\begin{array}{l}
\gamma \text { при } \omega_{2}=0, \\
\mu \text { при } \omega_{1}=0, \\
\gamma=\mu \text { при } \omega_{1} \neq 0, \omega_{2} \neq 0 .
\end{array}\right.
$$

Установлены следующие теоремы.

Те о ре м а 1. Пусть $\lambda_{1}-$ первое собственное число, $\vartheta_{1}(x)$-соответствующая первая собственная функция задачи (4), $\vartheta_{1}(x)$ удовлетворяет условию нормировки (5). Далее пусть начальная функция 
$u_{0}(x)$ такая, что $y_{0}=\int_{\Omega} \vartheta_{1}(x) \operatorname{Re} u_{0}(x) d x$ удовлетворяет условию

$$
y_{0}>\left(\frac{\lambda_{1}|\beta|}{\omega \rho}\right)^{\frac{1}{\rho}}
$$

где

$$
\stackrel{0}{\omega}=\left\{\begin{array}{l}
\omega e^{-\frac{\pi \alpha \rho}{2|\beta|}} n p u \alpha>0, \\
\omega \quad n p u \alpha \leq 0 .
\end{array}\right.
$$

Тогда максимальное время существования гладких решений задачи (1)-(3) оценивается следующей формулой:

$$
t_{\max }<\frac{1}{\lambda_{1}|\beta|} \arcsin \left(\frac{\lambda_{1}|\beta|}{\omega \rho y_{0}^{\rho}}\right) .
$$

Здесь $\omega$ определена формулой (6), $\rho-$ формулой (7).

Те орем а 2. Пусть $\lambda_{1}$ - первое собственное число, $\vartheta_{1}(x)$-соответствующая первая собственная функция задачи (4), $\vartheta_{1}(x)$ удовлетворяет условию нормировки (5). Далее пусть начальная функция $u_{0}(x)$ такая, что $y_{0}=\operatorname{sgn}(\beta) \int_{\Omega} \vartheta_{1}(x) \operatorname{Im} u_{0} d x$ удов-
летворяет условию

где

$$
y_{0}>\left(\frac{\lambda_{1}|\beta|}{2{ }^{0} \rho}\right)^{\frac{1}{\rho}}
$$

$$
\stackrel{0}{\omega}=\left\{\begin{array}{lc}
\omega e^{-\frac{\pi \alpha \rho}{|\beta|}} & n p u \alpha>0, \\
\omega & n p u \alpha \leq 0 .
\end{array}\right.
$$

Тогда максимальное время существования гладких решений задачи (1)-(3) оценивается следующей формулой:

$$
t_{\max }<\frac{1}{\lambda_{1}|\beta|} \arccos \left(1-\frac{\lambda_{1}|\beta|}{\omega \rho y_{0}^{\rho}}\right) .
$$

Здесь Ф определена формулой (6), $\rho-$ формулой (7).

3. Приведём схему доказательства теоремы 1. Введем на отрезке $[0, T]$, где $T=\frac{\pi}{2 \lambda_{1}|\beta|}$, функцию

$$
y_{1}(t)=\frac{1}{2} \int_{\Omega}\left[e^{\lambda_{1} z t} u(t, x)+e^{\lambda_{1} \bar{z} t} \bar{u}(t, x)\right] \vartheta_{1}(x) d x,
$$

где $z=\alpha+i \beta, \bar{z}=\alpha-i \beta, \bar{u}(x, t)-$ комплексно-сопряжённая функция к функции $u(x, t)$.

Справедлива следующая
Лемма 1. Пусть $u(x, t)$ - гладкое решение задачи (1)-(3), $\lambda_{1}-$ первое собственное число, $\vartheta_{1}(x)-$ соответствующая первая собственная функция задачи (4).

Тогда для любого $t \in[0, T]$ справедливо соотношение

$$
\frac{d y_{1}}{d t}=\cos \left(\lambda_{1} \beta t\right) I(t),
$$

где $I(t)=\int_{\Omega} f(u(x, t), \nabla u(x, t)) \vartheta_{1}(x) d x$.

Из леммы 1 при условии нормировки (5) на $\vartheta_{1}(x)$ выводится следующее дифференциальное неравенство первого порядка:

$$
\frac{d y_{1}}{d t} \geq \omega e^{-\lambda_{1} \alpha \rho t} \cos \left(\lambda_{1}|\beta| t\right) y_{1}^{1+\rho}
$$

для $\forall t \in[0, T]$, благодаря которому доказывается теорема 1.

4. Схема доказательства теоремы 2. $\underset{\text { функцию }}{\text { На отрезке }[0, T], \text { где } T=\frac{\pi}{\lambda_{1}|\beta|}, \text { введём }}$

$$
y_{2}(t)=-\frac{i}{2} \int_{\Omega}\left[e^{\lambda_{1} z t} u(x, t)-e^{\lambda_{1} \bar{z} t} \bar{u}(x, t)\right] \vartheta_{1}(x) d x .
$$

Лем ма 2. Пусть $и(x, t)-$ гладкое решение задачи (1)-(3), $\lambda_{1}-$ первое собственное число, $\vartheta_{1}(x)-$ соответствующая первое собственная функция задачи (1).

Тогда для $\forall t \in[0, T]$ справедливо соотношение

$$
\frac{d y_{2}}{d t}=\sin \left(\lambda_{1}|\beta| t\right) I(t),
$$

где $I(t)=\int_{\Omega} f(u, \nabla u) \vartheta_{1}(x) d x$.

Из леммы 2 для $y(t)=\operatorname{sgn}(\beta) y_{2}(t)$ выводится при условии нормировки (5) на функции $\vartheta_{1}(x)$ следующее дифференциальное неравенство первого порядка:

$$
\frac{d y}{d t} \geq \omega e^{-\lambda_{1} \alpha \rho t} \sin \left(\lambda_{1}|\beta| t\right) y^{1+\rho}
$$

на отрезке $[0, T]$, благодаря которому завершается доказательство теоремы 2.

Автор выражает искреннюю благодарность академику РАН В.П. Маслову за полезные советы и поддержку.

\section{СПИСОК ЛИТЕРАТУРЫ}

1. Rypdal K., Rasmussen J.J. // Phys. Scr. I. II. 1986. V. 33. P. 481-504.

ДОКЛАДЫ АКАДЕМИИ НАУК том 484 № 22019 
2. Захаров В.Е., Шабат А.Б. // ЖЭТФ. 1971. Т. 61. № 1. C. $118-134$.

3. Луговой В.И., Прохоров А.М. // УФН. 1973. Т. 111. № 11. С. 203-247.

4. Насибов Ш.М. // ДАН. 1985. Т. 285. № 4. С. 807-811.

5. Насибов Ш.М. // ДАН. 1989. Т. 304. № 2. С. 285-289.

6. Насибов Ш.М. // ДАН. 1989. Т. 307. № 3. С. 538-542.

7. Шабат А.Б. В кн.: Динамика сплошной среды. Новосибирск, 1969. В. 1. С. 180-194.

8. Кудряшов О.И. // Сиб. мат. журн. 1975. Т. 16. № 4. C. $866-868$.
9. Weinstein M. // Communs Partial and Different. Equats. 1986. V. 11. P. 545-565.

10. Nawa H. // Communs Pure and Appl. Math. 1999. V. 52. № 2. Р. 193-270.

11. Насибов Ш.М. // Дифференц. уравнения. 2003. Т. 39. № 8. C. 1087-1091.

12. Nasibov Sh.M. // J. Appl. Math. 2004. V. 1. P. 23-35.

13. Ginibre J., Velo G. // Physica D. 1996. V. 95. P. 191-238.

14. Ginibre J., Velo G. // Communs Math. Phys. 1997. V. 187. P. 45-79.

Владимиров В.С. Уравнения математической физики.

\section{ABSENCE OF GLOBAL SOLUTIONS OF A MIXED PROBLEM FOR A GINZBURG-LANDAU TYPE NONLINEAR EVOLUTION EQUATION}

\section{Sh. M. Nasibov}

Received by Academician of the RAS V. P. Maslov July 16, 2018

Received July 31, 2018

We study the problem of the absence of global solutions of the first mixed problem for one nonlinear evolution equation of Ginzburg-Landau type.We prove that global solutions of the studied problem are absent for "sufficiently large" values of the initial data.

Keywords: nonlinear evolution equation, Ginzburg-Landau type equation, mixed problem, global solution, absence of global solution. 